\title{
Brain Tumours: Rise in Glioblastoma Multiforme Incidence in England 1995-2015 Suggests an Adverse Environmental or Lifestyle Factor
}

\author{
Alasdair Philips (D), ${ }^{1,2}$ Denis L. Henshaw, ${ }^{1,3}$ Graham Lamburn, ${ }^{2}$ and Michael J. O'Carroll ${ }^{4}$ \\ ${ }^{1}$ Children with Cancer UK, 51 Great Ormond Street, London, WC1N 3JQ, UK \\ ${ }^{2}$ Powerwatch, Cambridgeshire, UK \\ ${ }^{3}$ Professor Emeritus, University of Bristol, UK \\ ${ }^{4}$ Professor Emeritus, Vice-Chancellor's Office, University of Sunderland, UK
}

Correspondence should be addressed to Alasdair Philips; alasdair@brambling.info

Received 19 December 2017; Revised 14 March 2018; Accepted 21 March 2018; Published 24 June 2018

Academic Editor: Evelyn O. Talbott

Copyright (C) 2018 Alasdair Philips et al. This is an open access article distributed under the Creative Commons Attribution License, which permits unrestricted use, distribution, and reproduction in any medium, provided the original work is properly cited.

\begin{abstract}
Objective. To investigate detailed trends in malignant brain tumour incidence over a recent time period. Methods. UK Office of National Statistics (ONS) data covering 81,135 ICD10 C71 brain tumours diagnosed in England (1995-2015) were used to calculate incidence rates (ASR) per 100k person-years, age-standardised to the European Standard Population (ESP-2013). Results. We report a sustained and highly statistically significant ASR rise in glioblastoma multiforme (GBM) across all ages. The ASR for GBM more than doubled from 2.4 to 5.0, with annual case numbers rising from 983 to 2531 . Overall, this rise is mostly hidden in the overall data by a reduced incidence of lower-grade tumours. Conclusions. The rise is of importance for clinical resources and brain tumour aetiology. The rise cannot be fully accounted for by promotion of lower-grade tumours, random chance or improvement in diagnostic techniques as it affects specific areas of the brain and only one type of brain tumour. Despite the large variation in case numbers by age, the percentage rise is similar across the age groups, which suggests widespread environmental or lifestyle factors may be responsible. This article reports incidence data trends and does not provide additional evidence for the role of any particular risk factor.
\end{abstract}

\section{Introduction}

The causes of brain tumours in adults remain largely unknown [1]. In 2011, the World Health Organisation (WHO) prioritised the monitoring of detailed brain tumour incidence trends through population-based cancer registries [2]. This article reports recent changes in malignant brain tumour incidence in England that include age, sex, morphology and tumour location.

\section{Materials and Methods}

2.1. Data. The International Classification of Diseases for Oncology (ICD-O) is a dual classification, with coding systems for both topography and morphology [3]. The relevant topology codes are listed in Table 1, along with the number of tumours diagnosed in 1995 and 2015.
There are 102 different ICD-O-3.1 morphology codes used in the data set, though many have few cases. The morphology code describes the cell type and its biological activity / tumour behaviour.

WHO last updated their classifications in 2016, but their changes have minimal impact on our analysis of the data [4, 5]. Malignant brain neoplasms without histology are recorded as ICD-10 D43 (D43.0 \& D43.2 supratentorial).

We used anonymised individual-level national cancer registration case data from the UK Office of National Statistics (ONS) for all 81,135 ICD10-C71 category primary malignant brain tumours diagnosed in England for the years from 1995 to 2015, plus 8,008 ICD10-D43 supratentorial malignant tumours without histology/morphology data from 1998-2015. The initial data is supplied by the National Cancer Registration Service (NCRS). The ONS then apply further 
TABLE 1: ONS WHO ICD10 brain tumour data for England.

\begin{tabular}{|c|c|c|c|}
\hline & & 1995 & 2015 \\
\hline C71 & Malignant primary neoplasm of brain & cases & cases \\
\hline C71.0 & Cerebrum except lobes \& ventricles & 154 & 213 \\
\hline C71.1 & Frontal lobe & 533 & 1231 \\
\hline C71.2 & Temporal lobe & 334 & 994 \\
\hline C71.3 & Parietal lobe & 506 & 587 \\
\hline C71.4 & Occipital lobe & 95 & 162 \\
\hline C71.5 & Cerebral ventricle & 31 & 47 \\
\hline C71.6 & Cerebellum & 138 & 143 \\
\hline C71.7 & Brain stem & 72 & 99 \\
\hline C71.8 & Overlapping lesion of brain & 262 & 208 \\
\hline C71.9 & Brain, unspecified site & 1286 & 770 \\
\hline C71 & All topology sites & 3411 & 4454 \\
\hline \multirow[t]{2}{*}{ D43 } & Uncertain behaviour (no histology data) & & \\
\hline & & 1998 & 2015 \\
\hline D43.0-43.2 & Unspecified tumour details - cases & 361 & 383 \\
\hline
\end{tabular}

validation checks and the UK Department of Health use the ONS data to inform policy making. The ONS state their cancer data are generally within $2 \%$ of the correct values [6]. Until about 2005, some cases in the oldest age-groups will not have been recorded in the cancer registries. Since 2005 this error is likely to be small.

Glioblastoma Multiforme (GBM), the most common and most malignant primary tumour of the brain, is associated with one of the worst five-year survival rates among all human cancers, with an average survival from diagnosis of only about 1 year. This ensures that few cases will be unrecorded in the ONS database and we show that their number of GBM tumours is similar to NHS hospital inpatient numbers. The data include the year of diagnosis, age at diagnosis, sex of patient, primary site and morphology code. National population estimates of age and gender by calendar year were also obtained from ONS data [7] and age-specific incidence rates per 100,000 person-years and for a wide variety of tumour types were calculated in 5-year age group bins for males and females separately.

Some published incidence analyses have used different criteria as to which glioma and astrocytoma should be considered malignant. WHO considers Grades I to IV as biologically malignant even if they have not been graded histologically malignant. We have taken the WHO/IARC morphology behaviour codes /3, /6 and / 9 as being histologically malignant which means that Grade I and II tumours are classed as low-grade malignancies.

We are not aware of any specific bias in the ONS data. There is a slight data-lag in cancer registry data, which are regularly checked and updated if necessary, but are generally stable after 3 to 5 years. Our ONS data extract is dated $4^{\text {th }}$ July 2017.

Brodbelt et al. (2015) [8] reported an analysis of treatment and survival for 10,743 GBM cases in England over the period 2007-2011, which had an overall median survival of only 6.1 months, rising to 14.9 months with maximal treatment.
Brodbelt et.al.s GBM case total from English hospital data is only $0.5 \%$ higher that our ONS GBM total of 10,687 cases for the same time period; this suggests that a very complete UK cancer diagnosis and registration system is now in place. In contrast, Ostrom et al. (2015) [9] reporting on USA SEER brain tumour data provide a scatter-plot that shows a median complete registration and histological confirmation level of only about $65 \%$, with the best examples returning less than $75 \%$ full completion in 2012 .

2.2. Confounding. We had a large number of categories and sub-categories in the data. It was necessary to combine some of these to increase the resolving power. We ran analyses separately for each site (C71.0 to C71.9), for each main type of tumour, and for tumour grade (I to IV). It was immediately obvious that the most significant change was in the incidence of GBM in frontal and temporal lobes. The obvious potential confounders would be the C71.8 (overlapping) and C71.9 (unspecified) categories due to better imaging techniques and we discuss this later.

2.3. Standardisation. Incidence rates rise dramatically with age and standardisation is necessary as population age profiles are changing with time. We calculated age-standardised incidence rates (ASR) per 100k person-years to the current recommended European Standard Population (ESP-2013), as it best represents the reality of the case burden on society [10]. Adjusting European cancer incidence to the World Standard Population is not helpful as the age-spectra are so different.

Table 2 lists the morphology codes with the highest case numbers, totalling 80354 tumours. Included in our analyses are an additional 781 cases in 78 other categories, each with fewer than 100 cases over the 21 years. A full listing of all the cases in the data set is provided in the Supplementary File [S1].

We needed to group data to improve resolution and reduce random data noise. We examined infant and child 
TABLE 2: ICD-O-3 morphology codes with more than 100 cases between 1995-2015 inclusive. (A full listing of all the morphology codes and cases is present in the Supplementary file).

\begin{tabular}{|c|c|c|c|c|c|}
\hline Morphology & Grade & All cases & Group & Sub-group & WHO/IARC summary description \\
\hline 80003 & 1 & 7776 & NOS & & unclassified, malignant, blastoma, NOS \\
\hline 80013 & 2 & 250 & carcinoma & & carcinoma, metastatic, NOS \\
\hline 80103 & 1 & 536 & carcinoma & & epithelial tumour, carcinoma, malignant \\
\hline 80106 & 2 & 281 & carcinoma & & carcinoma, metastatic, NOS \\
\hline 89633 & 2 & 131 & sarcoma & & rhabdoid sarcoma \\
\hline 90643 & 2 & 106 & & & germ cell neoplasia \\
\hline 93803 & 2 & 11269 & glioma & NOS & glioma, malignant, NOS, not neoplastic \\
\hline 93813 & 3 & 187 & glioma & astrocytic & gliomatosis cerebri \\
\hline 93823 & 2 & 1298 & glioma & astrocytic & mixed glioma / oligoastrocytoma \\
\hline 93913 & 2 & 1034 & glioma & ependymal & ependymoma \\
\hline 93923 & 3 & 313 & glioma & ependymal & anaplastic ependymoma \\
\hline 94003 & 2 & 7807 & glioma & astrocytic & astrocytoma, NOS, diffuse \\
\hline 94013 & 3 & 2832 & glioma & astrocytic & anaplastic astrocytoma (high grade) \\
\hline 94113 & 2 & 331 & glioma & astrocytic & germistocytic astrocytoma, diffuse \\
\hline 94203 & 2 & 420 & glioma & astrocytic & fibrillary astrocytoma, diffuse \\
\hline 94213 & 1 & 2125 & glioma & astrocytic & pilocytic astrocytoma \\
\hline 94243 & 2 & 106 & glioma & astrocytic & pleomorphic xantoastrocytoma \\
\hline 94403 & 4 & 37046 & glioma & GBM-IV & glioblastoma multiforme \\
\hline 94413 & 4 & 263 & glioma & GBM-IV & giant cell glioblastoma \\
\hline 94423 & 4 & 477 & glioma & GBM-IV & gliosarcoma \\
\hline 94503 & 2 & 2671 & glioma & oligodendrial & oligodendroglioma \\
\hline 94513 & 3 & 1339 & glioma & oligodendrial & anaplastic oligodendroglioma \\
\hline 94703 & 4 & 1178 & glioma & embryonal & medulloblastoma \\
\hline 94713 & 4 & 106 & glioma & embryonal & desmoplastic medulloblastoma \\
\hline 94733 & 4 & 472 & glioma & embryonal & primitive neuroectodermal tumour \\
\hline
\end{tabular}

neoplasms separately, but did not find any statistically significant time-trends. Three age-groups seemed reasonable. We chose a child, teenage and young-adult group (0-29), a main middle-age group (30-54) and an older age group (over 55 years of age). These reasonably split the population into three roughly equal (20, 18 and 16 million) groups of people. The case totals in the three groups were about $9.5 \mathrm{k}, 19.5 \mathrm{k}$ and $52 \mathrm{k}$ respectively. We tested moving the cut-point boundaries by 5 years in both directions and it made little difference to the overall results.

2.4. Analysis. The cases were analysed by morphology, topology, sex, age, age-specific and age-standardised incidence. The Annual Average Percentage Change (AAPC) and corresponding 95\% CI and $\mathrm{p}$-values were calculated using Stata SE12.1 (StataCorp). A linear model on the log of the age-standardised rates, which tests for a constant rate of change $\left(\mathrm{e}^{(\ln (\mathrm{rate}))}\right)$, best fitted the data. See Supplementary File sections [S2] and [S3].

2.5. Background. In a major 2013 review article, Hiroko Ohgaki and Paul Kleihues [11] wrote "Glioblastoma is the most frequent and malignant brain tumor. The vast majority of glioblastomas ( $90 \%)$ develop rapidly de novo in elderly patients, without clinical or histologic evidence of a less malignant precursor lesion (primary glioblastomas). Secondary glioblastomas progress from low-grade diffuse astrocytoma or anaplastic astrocytoma. They manifest in younger patients, have a lesser degree of necrosis, are preferentially located in the frontal lobe, and carry a significantly better prognosis."

Overall primary malignant brain tumour ASRs are only rising slowly and are often considered fairly static. Figure 1 shows the age-standardised trends from 1971 to 2015. From the 1970s to about 2000 there was a fairly steady rise in recorded overall incidence, however since then the rise has slowed, though clinicians have been reporting a rise in highgrade, aggressive tumours.

Overall adult survival for all malignant brain tumours after diagnosis during 2006-2010 was about 35\% for one year and $15 \%$ for five years, falling to about $3 \%$ for aggressive grades-III and IV tumours. ONS data show agestandardised death rates from malignant brain tumours (C71) have increased by 7\% between 2001 and 2015, showing that improvements in treatment alone are inadequate and that there is a need to find ways of preventing brain cancer [12].

\section{Results}

Comparing new case numbers in 2015 with 1995 shows an extra 1548 aggressive GBM tumour cases annually. Figure 2 


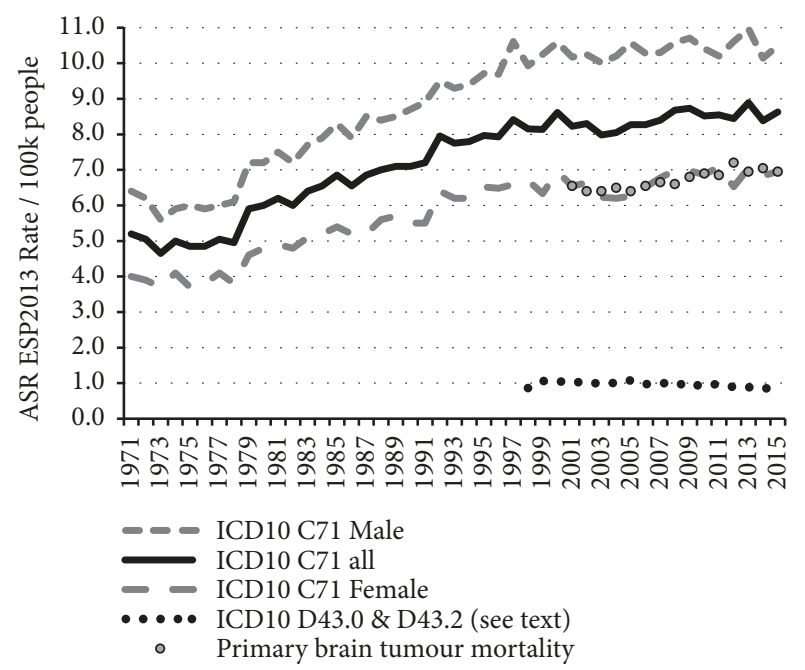

FIgURE 1: Age-standardised overall trends from 1971 to 2015 using data in ONS MB1 series, including a smaller number of supratentorial neoplasms without histology or morphology data coded D43.0 \& D43.2. The data table for this figure is in the SI file as [S4].

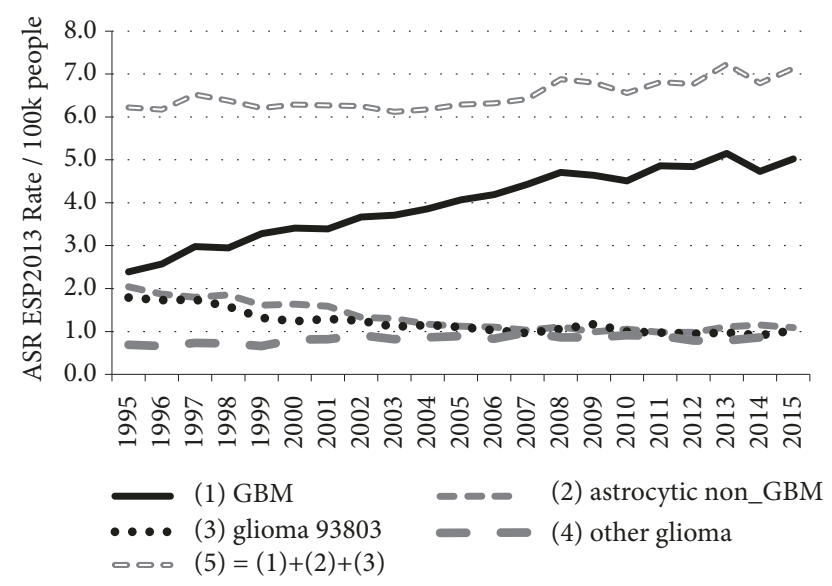

FIGURE 2: Age-standardised incidence rates for all C71 glioma cases diagnosed between 1995 and 2015 analysed by type and year (Data in Table 3). Grouping details: $(1)=94403-94433(2)=$ 93843, 94003-94303 (3) = $93803(4)=93813,93823,93903-93943$, 94503-94733.

and Table 3 show that up to about 2004 the overall rise in GBM incidence (Annual Average Percentage Change (AAPC) 5.2\%, 95\% CI 3.7-6.6, p < 0.00003) could be mostly compensated for by the fall in incidence of all lower grade astrocytoma and "glioma, malignant, NOS, ICD10-93803". This leaves a fairly steady rise in the GBM ASR from 2004 to 2015 (AAPC $2.2 \%, 95 \%$ CI 1.4-3.0, p < 0.0001).

Ohgaki and Kleihues [11] reported that most secondary GBMs are found in younger middle-age people and most primary GBMs are in over 60s. We tested our (30-54) and $(>54)$ age group data, splitting the total GBM into de novo and promoted tumours. We estimated the maximum possible number of promoted tumours using the change in the grades
II and III diffuse and anaplastic astrocytomas. The results are shown in Figures 3(a) and 3(b). These are discussed later.

We found a large decrease of ASR over time for Grade-II diffuse astrocytoma, a slight rise in ASR for WHO Grade-III anaplastic astrocytoma (94013; 2832 cases). There was little change in rates of anaplastic oligodendroglioma (94513; 1339 cases), anaplastic ependymoma (93923; 313 cases) Grade-II oligodendroglioma (94503; 2671cases), embryonal, or ependymal tumours.

Figure 4 shows the relative increase in age-specific GBM incidence between the averaged periods (1995-1999) and (2011-2015) for 5-year age-groups. This 1.5-fold change is remarkably similar across the age-groups, suggesting a universal factor.

Figure 5 shows ASR GBM rates for frontal lobe, temporal lobe, unspecified \& overlapping (C71.8 \& C71.9) and 'all other brain regions'. Most of the rise is in the frontal and temporal lobes, and most of the cases are in people over 55 years of age, with a highly statistically significant overall AAPC of 7.6\% (see Table 4). There was an extra rise in frontal and temporal GBM incidence between 2006 and 2008, which coincided with a slight reduction in the GBM ASR in overlapping and unspecified regions and may be due to improved imaging.

\section{Discussion}

Using sufficiently high-quality data, we present a clearer picture of the changing pattern in incidence of brain tumour types than any previously published. We report a sustained and highly statistically significant ASR rise in GBM across all ages and throughout the 21 years (1995-2015), which is of importance both for clinical resources and brain tumour aetiology.

Dobes et al. (2011) [13] reported a significant increase in malignant tumour incidence from 2000 to 2008 in the $\geq 65$-year age group. In a second article they noted an increasing incidence of GBM (APC, 3.0; 95\% CI, 0.5-5.6) in patients in the same age group, especially in temporal and frontal lobes [14]. De Vocht et al. (2011) [15] reported a rise in temporal lobe tumour incidence in ONS data, but dismissed its significance. In a 2016 paper he claimed no increase in GBM incidence, but later published a major correction to the paper that shows an increase [16].

Zada et al. (2012) [17] using USA SEER data for 1992-2006 reported a rising trend in frontal and temporal lobe tumours, the majority of which were GBM, with a decreased incidence of tumours across all other anatomical sub-sites. Ho et al. (2014) [18] reported a 2.2-fold increase in glioblastoma incidence in the Netherlands over the period 1989-2010 (APC $3.1, \mathrm{p}<0.001)$.

There were no material classification changes over the analysis period that might explain our findings [19], though multidisciplinary team working was strengthened (2005 onwards) and better imaging has resulted in improved diagnosis along with a more complete registration of brain tumours in the elderly. We analysed our data in 5-year age group categories to look for evidence of improved diagnosis; the data do suggest diagnosis and registration have improved 


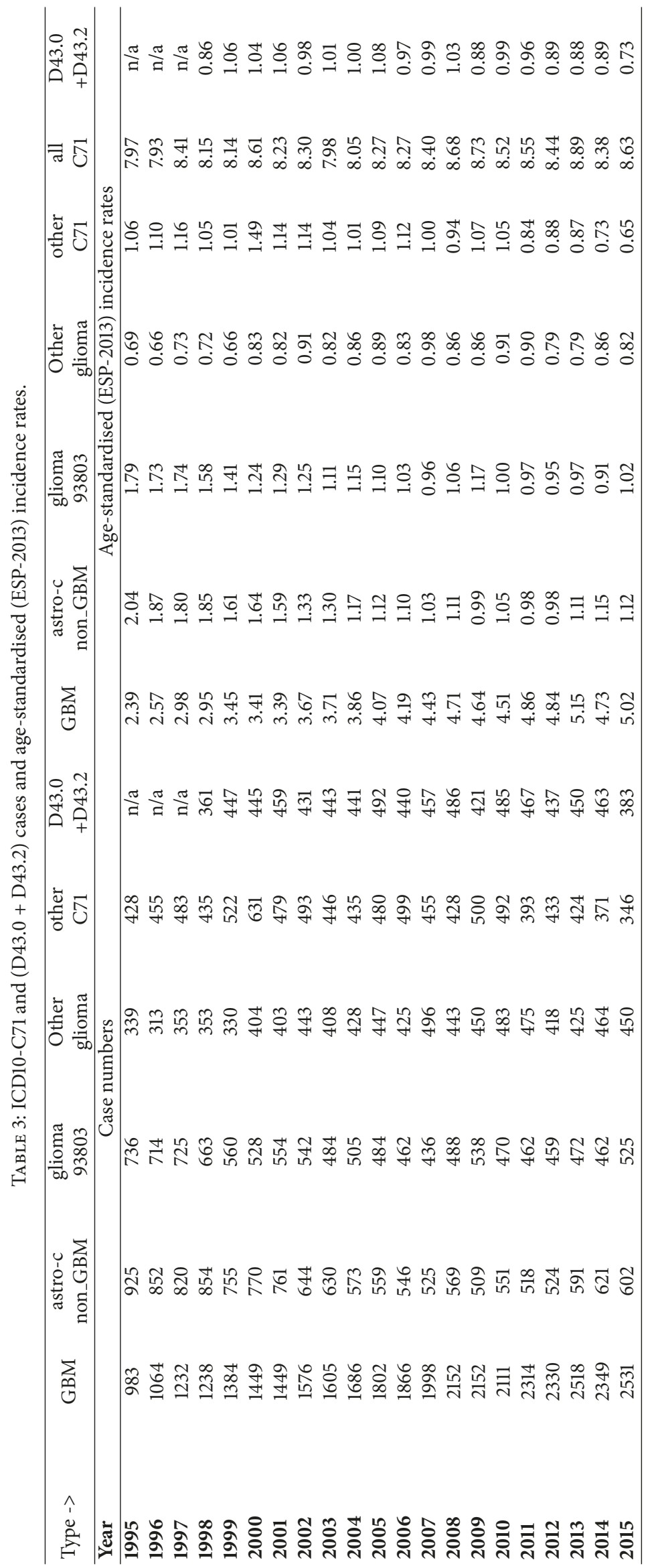




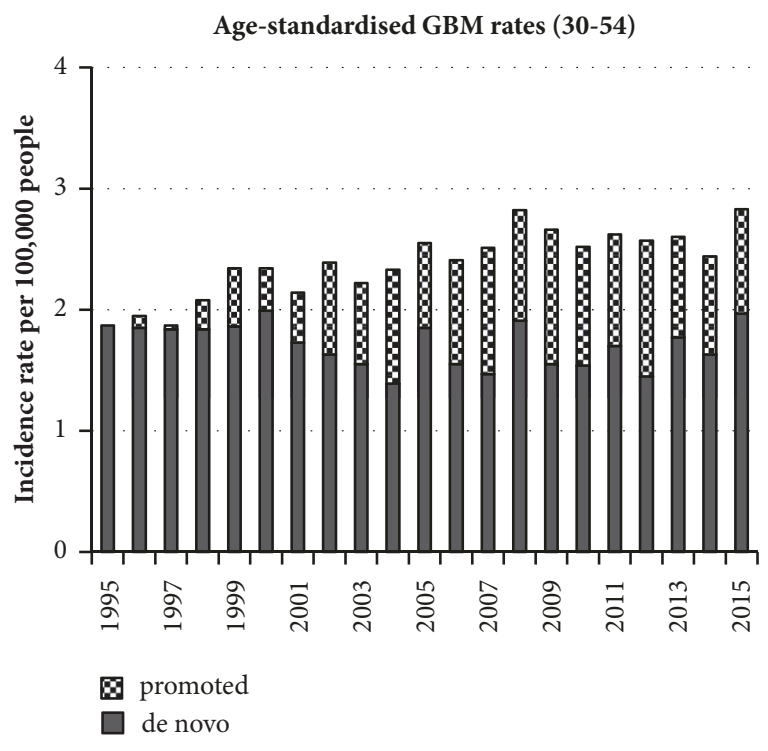

(a)

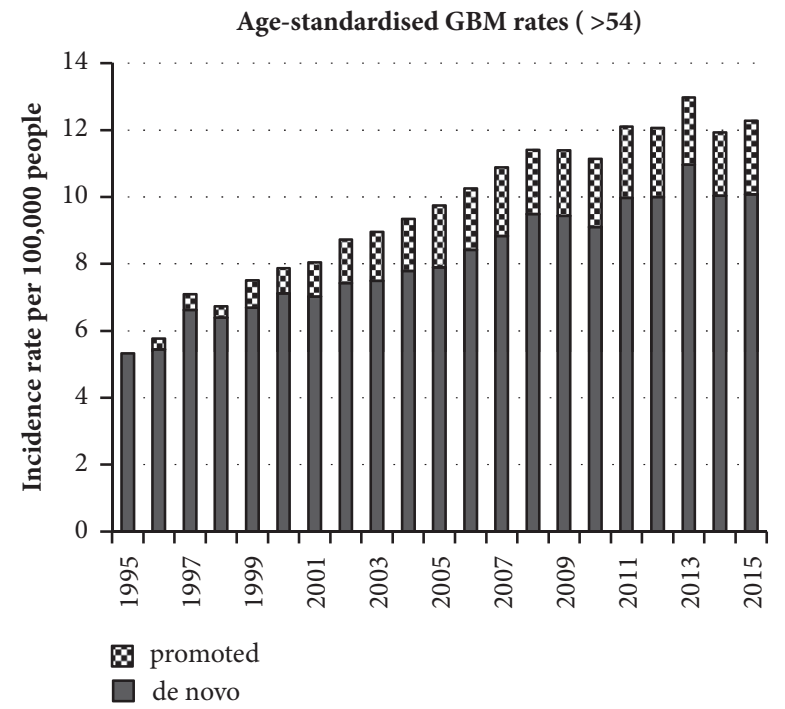

(b)

FIGURE 3: Age-standardised rates for two age groups. The possible split between de novo and secondary promoted GBMs is based on incidence change of Grades II and III diffuse and anaplastic astrocytoma.

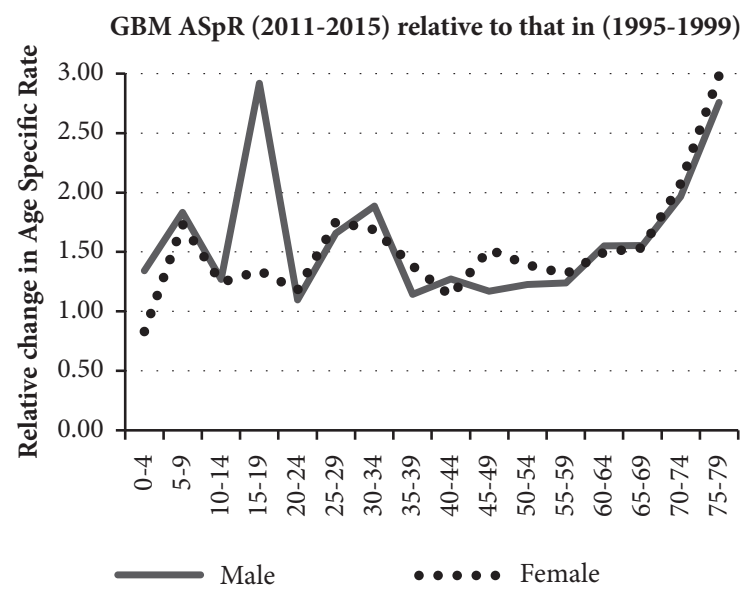

FIGURE 4: Relative change in GBM age-specific incidence rates (ASpR) averaged over two five-year periods 1995-1999 and 2011-2015 in 5-year age bands and gender.

in people aged over 70 . However, at earlier ages the incidence rate of 'all' glioma (and all C71) registrations have remained almost constant, whereas the rates for lower-grade tumours fell until about 2006 and have since remained fairly static as the rate for GBM has risen steadily.

Most GBM cases seem to originate without any known genetic predisposition. GBMs from promoted lower-grade gliomas usually have different molecular genetic markers from de novo GBMs [20]. The 2016 revision of the WHO classification of CNS tumours $[3,4]$ highlights the need for recording molecular genetic markers and divides glioblastomas into two main groups. The IDH-wildtype mostly corresponds to clinically defined primary or de novo glioblastoma and accounts for about $90 \%$ of cases. The remaining

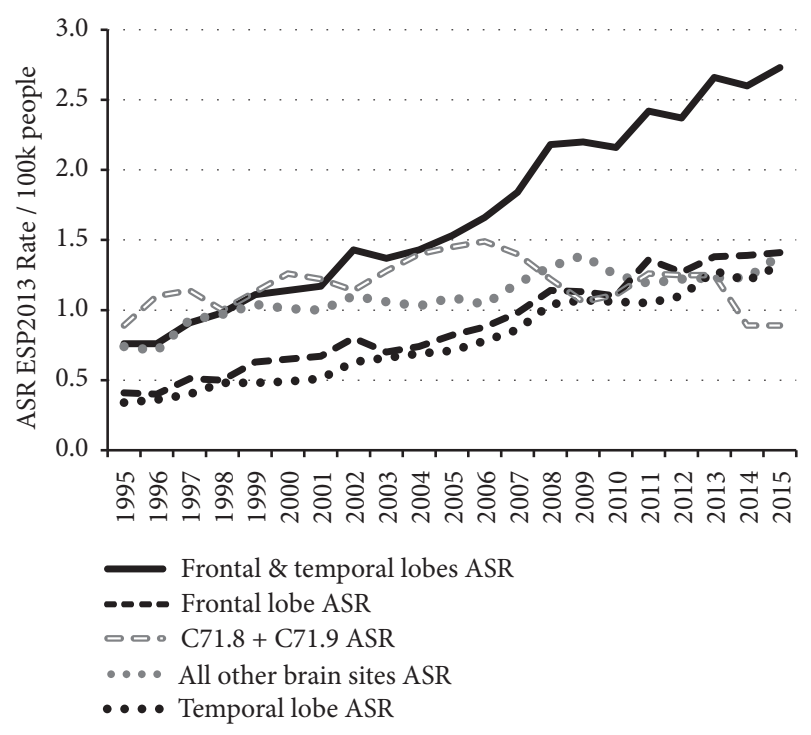

FIGURE 5: Frontal and temporal lobe GBM age-standardised incidence rates by tumour site and year (data table in the SI as [S6]).

$10 \%$ are IDH-mutant cases, which usually arise in younger patients and mostly correspond to secondary or promoted lower-grade diffuse glioma [11, 21]. Figures 3(a) and 3(b) support the conclusion of Ohgaki and Kleihues [11] that promoted (secondary) tumours mainly occur in younger people and that de novo GBMs dominate in the over-54 age group. It is important that this pattern is monitored using modern genetic techniques.

GBM tumours are almost always fatal and are not likely to have been undiagnosed in the time-frame of our data. It is possible that some elderly cases were not fully classified, 


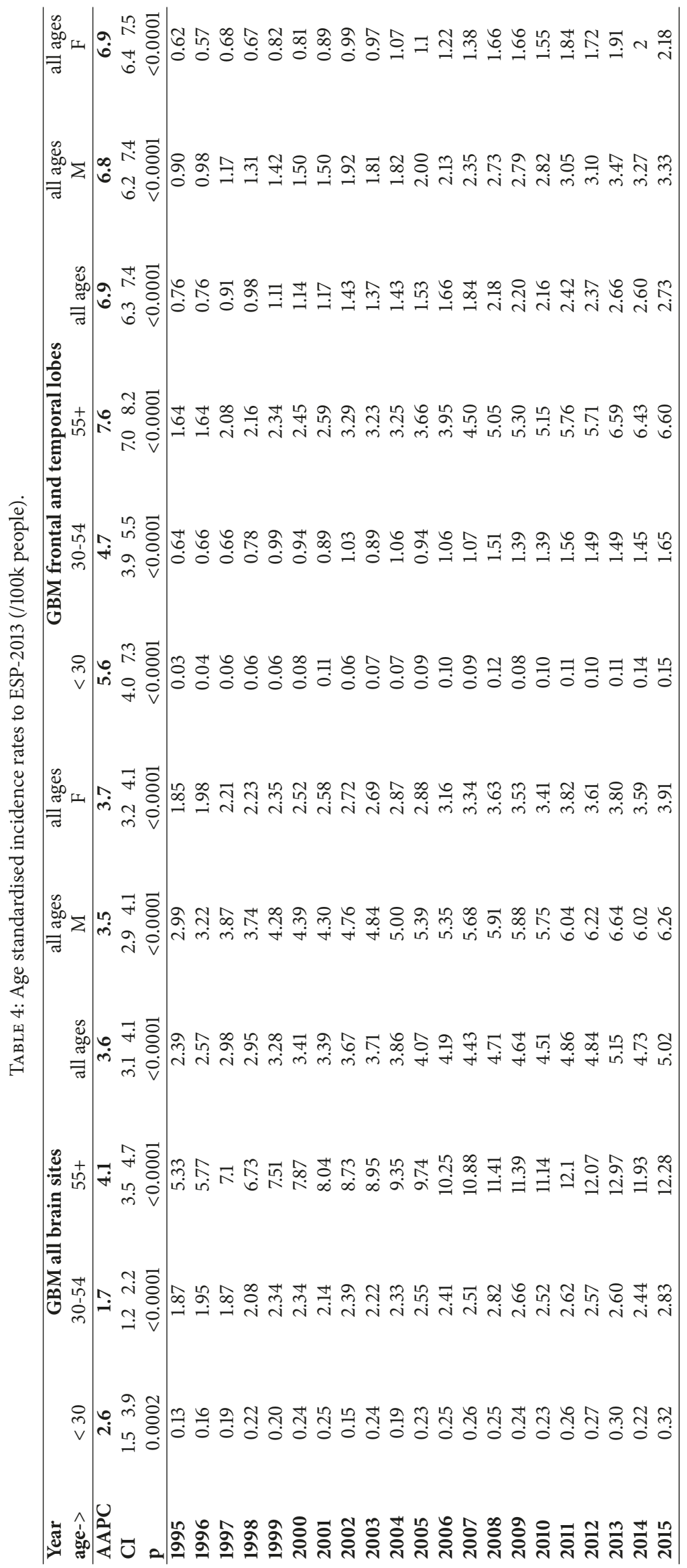


but then they should have been recorded as ICD10-D43. However, as D43 rates have remained very constant over this time period (see Figure 1), this is unlikely to have been a significant confounder.

4.1. Possible Causal Factors. We cite examples of some possible causal factors that have been discussed in the literature that could contribute changes in GBM incidence. In an important 2014 "state of science" review of glioma epidemiology, Ostrom et al. [22] list and discuss a number of potential factors that have been associated with glioma incidence, some of which we list below.

Ionising radiation, especially from X-rays used in CT scans, has the most supportive evidence as a causal factor. Due to the easy availability of CT imaging and relative lack and higher cost of MRI imaging in UK NHS hospitals, CT scans are often used, especially for initial investigations. Their use over the period 1995-2013 is shown in the Supplementary File [S6]. Given the time-frame of the trend that we have identified, we suggest that $\mathrm{CT}$ imaging X-ray exposures should be further investigated for both the promotion and initiation of the rising incidence of GBM tumours that we have identified.

Preston et al. (2007) [23] concluded that radiationassociated cancer persists throughout life regardless of age at exposure and that glioma incidence shows a statistically significant dose response. Our oldest age group also experienced atmospheric atomic bomb testing fallout and some association with ingested and inhaled radionuclides should not be dismissed as a possible factor. England was in one of the highest exposed regions for atmospheric testing fallout as determined by the United Nations Scientific Committee on the Effects of Atomic Radiation, UNSCEAR 2000 Report [24]. Further information is given in Supplementary File S7. If only some of the population were susceptible and received a significant dose, any resulting extra cancers would show up in the ONS data.

The European Study of Cohorts for Air Pollution Effects by Andersen et al. (2017) [25] found suggestive evidence of an association between traffic-related air pollution and malignant brain tumours.

There is increasing evidence literature that many cancers including glioma have a metabolic driver due to mitochondrial dysfunction resulting in downstream genetic changes in the nucleus [26-28].

The International Agency for Research on Cancer (IARC) judged both power-frequency ELF (2002) [29] and radio-frequency RF (2011) [30] electromagnetic fields as Group 2B 'possible human carcinogens'. Villeneuve et al. (2002) [31] concluded that occupational (ELF) magnetic field exposure increases the risk of GBM with an OR $=5.36$ (95\% CI: 1.2 - 24.8). Hardell and Carlberg (2015) [32] have reported an increase in high-grade glioma associated with mobile phone use. The multi-country Interphone study [33] collected data from 2000 to 2003 and included few people over 55 years of age and would have been unable to resolve any association involving older-aged people. Volkow et al. (2011) [34] found that, in healthy participants and compared with no exposure, a 50-minute cell phone exposure produced a statistically significant increase in brain glucose metabolism in the orbitofrontal cortex and temporal pole regions closest to the handset.

\section{Conclusions}

(1) We show a linear, large and highly statistically significant increase in primary GBM tumours over 21 years from 1995-2015, especially in frontal and temporal lobes of the brain. This has aetiological and resource implications.

(2) Although most of the cases are in the group over 54 years of age, the age-standardised AAPC rise is strongly statistically significant in all our three main analysis age groups.

(3) The rise in age-standardised incidence cannot be fully accounted for by improved diagnosis, as it affects specific areas of the brain and just one type of brain tumour that is generally fatal. We suggest that widespread environmental or lifestyle factors may be responsible, although these results do not provide additional evidence for the role of any particular risk factor.

(4) Our results highlight an urgent need for funding more research into the initiation and promotion of GBM tumours. This should include the use of CT imaging for diagnosis and also modern lifestyle factors that may affect tumour metabolism.

\section{Data Availability}

The data were obtained from the UK Office for National Statistics (ONS), who are the legal owners of the data. Some data are publicly available in the ONS annual MB1 data series, which are freely downloadable from the ONS website, but this article uses the latest updated data, plus ICD-O-3 morphology codes, extracted under personal researcher contract from the ONS database in July 2017. ONS Data Guardian approval was required for the supply, control and use of the data. A nominal charge is made by the ONS for such data extraction. We are not permitted to supply the raw ONS extracted data to anyone else. Other researchers can obtain the latest data directly from the ONS in a similar manner. The authors provide some extra tables and figures in the Supplementary File downloadable from the journal website. Supplementary data can be obtained from the corresponding author upon request.

\section{Conflicts of Interest}

Alasdair Philips: Independent Engineer and Scientist. (a) Trustee of Children with Cancer UK (unpaid); (b) On a voluntary unpaid basis, has run Powerwatch for 25 years (a small UK NGO providing free information on possible health associations with EMF/RF exposure); (c) Technical Director 
and shareholder of EMFields Solutions Ltd., who design and sell EMF/RF measuring instruments and protective shielding items; (d) Shareholder of Sensory Perspective Ltd.; (e) Occasional voluntary advisor to the Radiation Research Trust (Registered Charity). Denis L. Henshaw: (a) Scientific Director of Children with Cancer UK (honorarium basis); (b) Shareholder of Track Analysis Systems Ltd., a company offering radon measurement services; (c) Voluntary scientific advisor for Electrosensitivity UK (Registered Charity). Michael J. O'Carroll: (a) Chairman of Rural England against Overhead Line Transmission group; (b) Occasional advisor to the Radiation Research Trust. Graham Lamburn: (a) Acts as voluntary unpaid 'Technical Manager' for Powerwatch.

\section{Authors' Contributions}

Alasdair Philips and Graham Lamburn conceived the study and first-drafted most of the manuscript with significant input from Denis L. Henshaw and Michael J. O'Carroll. Graham Lamburn organised the data obtained from the UK ONS and wrote the database analysis scripts. All authors had full access to the results of all analyses and have provided strategic input over several years of following the ONS brain tumour data. All authors have approved the final manuscript. Alasdair Philips is the guarantor for the ONS data.

\section{Funding}

This research received no funding from any external agency or body. The ONS data extracts were paid for personally by Alasdair Philips. Administration costs were paid for personally by the authors.

\section{Acknowledgments}

We are very grateful to Professor Geoffrey Pilkington and Professor Annie Sasco for their invaluable comments on early drafts of this paper. We thank the ONS for providing the data and Michael Carlberg, MSc for advice regarding statistical analysis.

\section{Supplementary Materials}

S1. Table of data morphology coding and the case numbers used in the study. S2. GBM case numbers and age-specific incidence rate data used in the study. S3. Sample STATA data and DO script. S4. Data table for Figure 1. S5. Data table for Figure 5. S6. CT and MRI use in the UK NHS. S7. Some notes on atomic bomb testing and other nuclear fallout in England. (Supplementary Materials)

\section{References}

[1] M. L. Bondy, M. E. Scheurer, B. Malmer et al., "Brain tumor epidemiology: Consensus from the Brain Tumor Epidemiology Consortium," Cancer, vol. 113, no. 7, pp. 1953-1968, 2008.

[2] E. Van Deventer, E. Van Rongen, and R. Saunders, "WHO research agenda for radiofrequency fields," Bioelectromagnetics, vol. 32, no. 5, pp. 417-421, 2011.
[3] "IARC - International Classification of Diseases of Oncology ICD-O-3," http://codes.iarc.fr/abouticdo.php.

[4] D. N. Louis, A. Perry, G. Reifenberger et al., "The 2016 World Health Organization Classification of Tumors of the Central Nervous System: a summary," Acta Neuropathologica, vol. 131, no. 6, pp. 803-820, 2016.

[5] D. N. Louis, H. Ohgaki, O. D. Wiestler et al., "WHO Classification of Tumours of the Central Nervous System. 4th (rev)," in IARC, ISBN-10 9283244923, 2016.

[6] UK Office for National Statistics, "Cancer Statistics: Registrations Series MB1," 2017, https://www.ons.gov.uk/peoplepopulationandcommunity/healthandsocialcare/conditionsanddiseases/ bulletins/cancerregistrationstatisticsengland/2015\#data-quality.

[7] UK Office for National Statistics, "Population Estimates for UK, England and Wales, Scotland and Northern Ireland," 2017, https:// www.ons.gov.uk/peoplepopulationandcommunity/populationandmigration/populationestimates.

[8] A. Brodbelt, D. Greenberg, T. Winters, M. Williams, S. Vernon, and V. P. Collins, "Glioblastoma in England: 2007-2011," European Journal of Cancer, vol. 51, no. 4, pp. 533-542, 2015.

[9] Q. T. Ostrom, H. Gittleman, J. Fulop et al., "CBTRUS statistical report: primary brain and central nervous system tumors diagnosed in the united states in 2008-2012," Neuro-Oncology, vol. 17, Supplement 4, pp. iv1-iv62, 2015.

[10] European Union, "European Standard Population," http://ec .europa.eu/eurostat/en/web/products-manuals-and-guidelines/ -/KS-RA-13-028.

[11] H. Ohgaki and P. Kleihues, "The definition of primary and secondary glioblastoma," Clinical Cancer Research, vol. 19, no. 4, pp. 764-772, 2013.

[12] UK Office for National Statistics, " $<1971-1994$ 8290769_tcm77395904.xls>," downloaded from the ONS, 26th September, and for 1995-2013 data, Table 13 in https://www.ons.gov.uk/file?uri=/ peoplepopulationandcommunity/healthandsocialcare/conditionsanddiseases/datasets/cancerregistrationstatisticscancerregistrationstatisticsengland/2015/cancerregistrations2015final22.05.2017.xls downloaded from the ONS, 10th July 2017.

[13] M. Dobes, V. G. Khurana, and B. Shadbolt, "Increasing incidence of glioblastoma multiforme and meningioma, and decreasing incidence of Schwannoma (2000-2008): findings of a multicenter Australian study," Surgical Neurology International, vol. 2, no. 176, pp. 1-7, 2011.

[14] M. Dobes, B. Shadbolt, V. G. Khurana et al., "A multicenter study of primary brain tumor incidence in Australia (2000-2008)," Neuro-Oncology, vol. 13, no. 7, pp. 783-790, 2011.

[15] F. De Vocht, "Inferring the 1985-2014 impact of mobile phone use on selected brain cancer subtypes using Bayesian structural time series and synthetic controls," Environment International, vol. 97, pp. 100-107, 2016.

[16] F. De Vocht, "Corrigendum to "Inferring the 1985-2014 impact of mobile phone use on selected brain cancer subtypes using Bayesian structural time series and synthetic controls. [Environ. Int. (2016), 97, 100-107]," Environment International, vol. 101, pp. 201-202, 2017, http://www.sciencedirect.com/science/article/pii/ S0160412017301241.

[17] G. Zada, A. E. Bond, Y.-P. Wang, S. L. Giannotta, and D. Deapen, "Incidence trends in the anatomic location of primary malignant brain tumors in the United States: 1992-2006," World Neurosurgery, vol. 77, no. 3-4, pp. 518-524, 2012.

[18] V. K. Y. Ho, J. C. Reijneveld, R. H. Enting et al., "Changing incidence and improved survival of gliomas," European Journal of Cancer, vol. 50, no. 13, pp. 2309-2318, 2014. 
[20] G. P. Dunn, M. L. Rinne, and J. Wykosky, "Emerging insights into the molecular and cellular basis of glioblastoma," Genes \& Development, vol. 26, pp. 756-784, 2012.

[21] H. Ohgaki and P. Kleihues, "Genetic alterations and signaling pathways in the evolution of gliomas," Cancer Science, vol. 100, no. 12, pp. 2235-2241, 2009.

[22] Q. T. Ostrom, L. Bauchet, F. G. Davis et al., "The epidemiology of glioma in adults: A state of the science review," Neuro-Oncology, vol. 16, no. 7, pp. 896-913, 2014.

[23] D. L. Preston, E. Ron, S. Tokuoka et al., "Solid cancer incidence in atomic bomb survivors:1958-1998," Radiation Research, vol. 168, no. 1, pp. 1-64, 2007.

[24] United Nations Scientific Committee on the Effects of Atomic Radiation, UNSCEAR 2000 Report to the General Assembly, United Nations, New York, NY, USA, 2000.

[25] Z. J. Andersen, M. Pedersen, G. Weinmayr et al., "Longterm exposure to ambient air pollution and incidence of brain tumor: the European Study of Cohorts for Air Pollution Effects (ESCAPE)," Neuro-Oncology, vol. 20, no. 3, pp. 420-432, 2017.

[26] T. N. Seyfried, "Cancer as a mitochondrial metabolic disease," Frontiers in Cell and Developmental Biology, vol. 3, 43 pages, 2015.

[27] M. G. Abdelwahab, K. E. Fenton, M. C. Preul et al., "The ketogenic diet is an effective adjuvant to radiation therapy for the treatment of malignant glioma," PLoS ONE, vol. 7, no. 5, Article ID e36197, 2012.

[28] T. N. Seyfried, R. E. Flores, A. M. Poff, and D. P. D’Agostino, "Cancer as a metabolic disease: implications for novel therapeutics," Carcinogenesis, vol. 35, no. 3, pp. 515-527, 2014.

[29] IARC, Monographs on the Evaluation of Carcinogenic Risks to Humans, Non-Ionizing Radiation, Part 1: Static and Extremely Low-Frequency (ELF) Electric and Magnetic Fields, vol. 80, 2002.

[30] IARC, Monographs on the Evaluation of Carcinogenic Risks to Humans, NonIonizing Radiation, Part 2: Radiofrequency Electromagnetic Fields, vol. 102, 2013.

[31] P. J. Villeneuve, D. A. Agnew, K. C. Johnson et al., "Brain cancer and occupational exposure to magnetic fields among men: Results from a Canadian population-based case-control study," International Journal of Epidemiology, vol. 31, no. 1, pp. 210-217, 2002.

[32] L. Hardell and M. Carlberg, "Mobile phone and cordless phone use and the risk for glioma-analysis of pooled case-control studies in Sweden, 1997-2003 and 2007-2009," Pathophysiology, vol. 22, no. 1, pp. 1-13, 2015.

[33] C. Wild, IARC Report to the Union for International Cancer Control (UICC) on the Interphone Study, WHO, IARC, Lyon, France, 03 October 2011.

[34] N. D. Volkow, D. Tomasi, G.-J. Wang et al., "Effects of cell phone radiofrequency signal exposure on brain glucose metabolism," The Journal of the American Medical Association, vol. 305, no. 8 , pp. 808-813, 2011. 


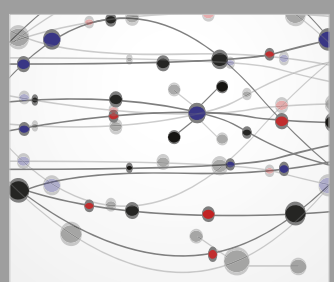

The Scientific World Journal
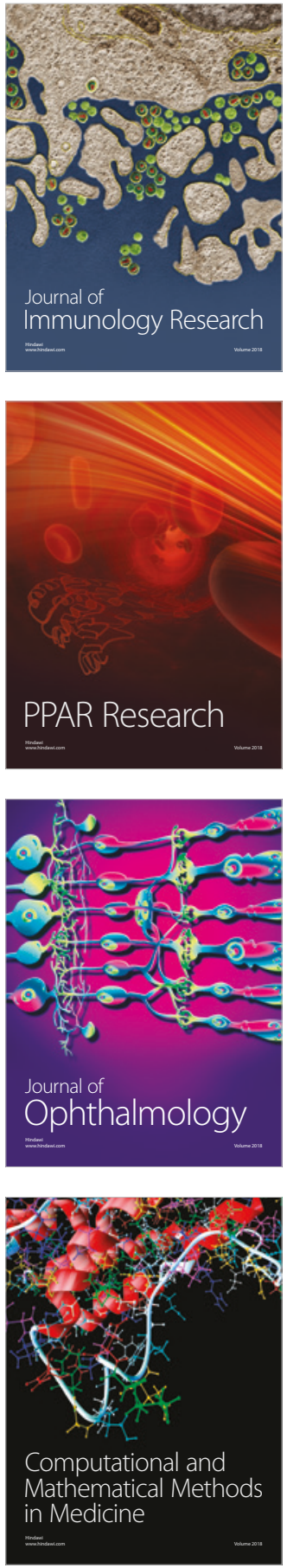

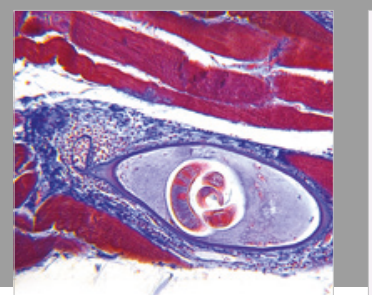

Gastroenterology Research and Practice

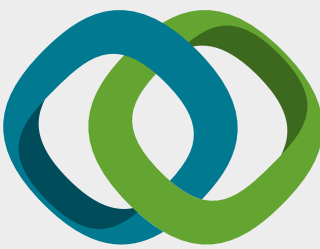

\section{Hindawi}

Submit your manuscripts at

www.hindawi.com
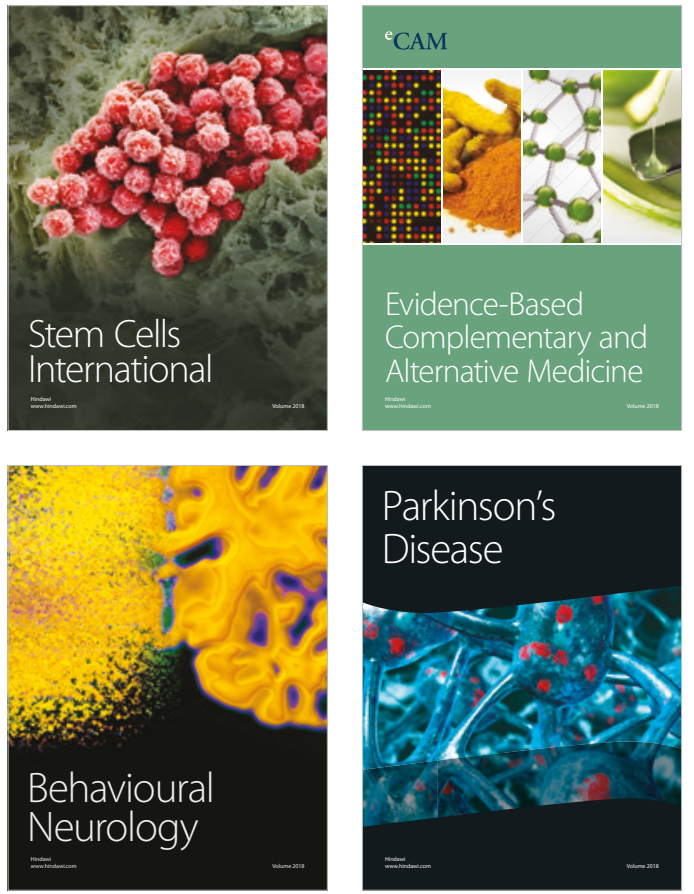

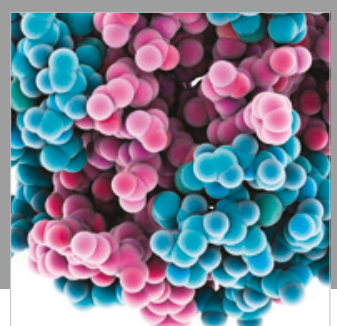

ournal of

Diabetes Research

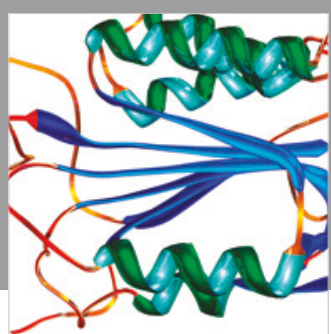

Disease Markers
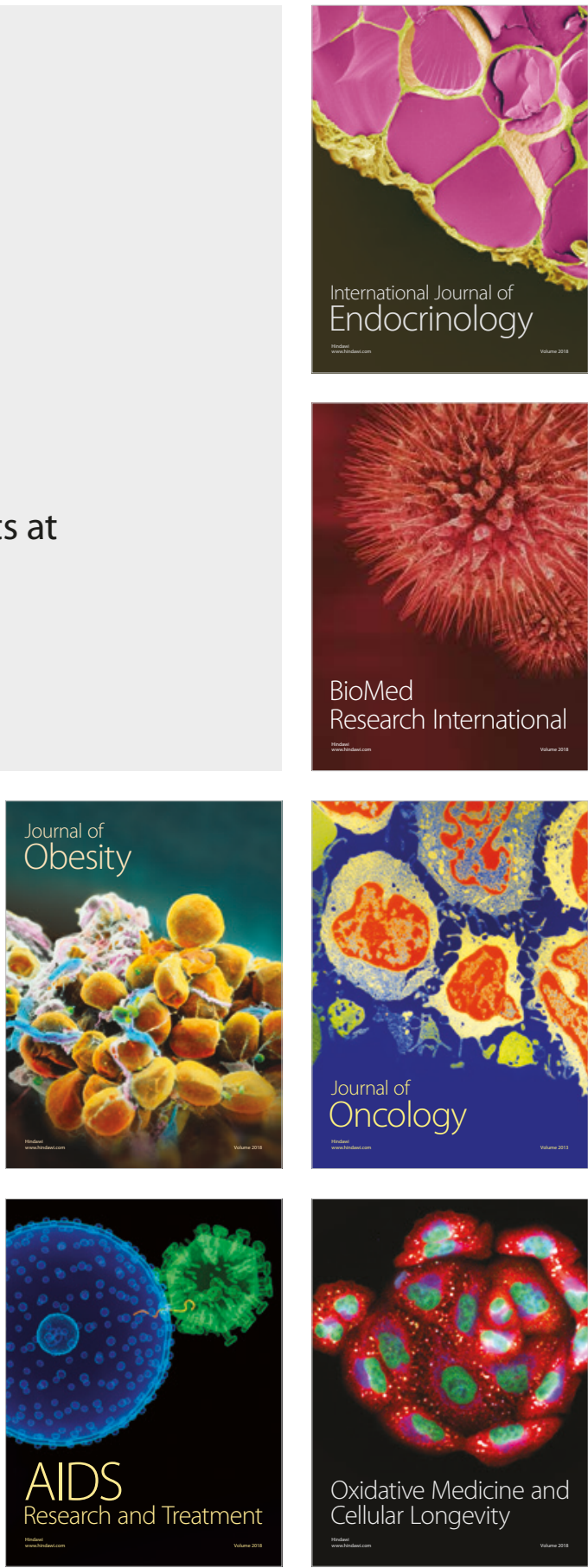